\title{
5-Alpha-Reductase 2 Deficiency in Newborns: A Review
}

\author{
Narasimha Prasad Vijayashankar', Artem Artemev², Anastasia Pougno², Gopikumar MS ${ }^{3}$ \\ ${ }^{1}$ Assistant Professor, Department of Pharmacology, Xavier University School of Medicine, Aruba, ${ }^{2}$ Basic Science \\ Students, Xavier University School of Medicine, Aruba, ${ }^{3}$ Associate Professor, Department of Physiology, Xavier \\ University School of Medicine, Aruba
}

\begin{abstract}
Disorders of sex development (DSD) are mostly associated with gender ambiguity at birth and poses a real challenge in diagnosis. 5 alpha reductase 2 deficiency (5AR2D) is one such DSD associated with deficiency of a key male sex hormone called as Dihydrotestosterone (DHT) due to lack of 5 alpha reductase type 2 enzyme which converts testosterone to Dihydrotestosterone. This hormone is very vital for the development of external genitalia during embryogenesis, and the deficiency leads to partial or abnormal male external genitalia in the affected neonate. 5AR2D is due to mutation in steroid-5- alpha-reductase, alpha polypeptide 2 (SRD5A2) genes on chromosome 2, band p23. Clinical presentation of these children vary from normal male genitalia to a completely female genitalia. 5AR2D is associated with deficiency of DHT and normal or increased testosterone levels. Most of the children with 5AR2D are raised as females and gender conversion to male happens at around puberty due to masculine and voice changes caused by testosterone. Early diagnosis is beneficial in treating this problem. Elevated testosterone: DHT ratio with administration of beta human chorionic Gonadotropin (HCG) is the gold standard test in diagnosis of this defect. Most patients of 5 alpha reductase 2 deficiency are considered infertile, but with recent advancements in assisted reproductive techniques a few 5AR2D have been successful in giving birth to their offsprings.
\end{abstract}

Key words: Disorders of sex development (DSD), 5 alpha reductase deficiency, Di-hydro-testosterone (DHT)

\section{Introduction}

DSD are a group of pediatric disorders associated with inconformity of gonads, chromosomes and hence external genitalia. DSD can be of two types based on the chromosomal status as 46, XX DSD and 46, XY DSD. In 46, XY DSD the primary sex organ is most of the affected individuals is testes, but associated with a deficiency in sex hormones or abnormal response of the individuals to the sex hormones. In majority of 46, XY $\mathrm{DSD}$, if the $\mathrm{Y}$ chromosome is normal and the primary sex organ is tests, then the major mutations are seen in either androgen receptor or in SRD5A2 genes. SRD5A2 genes regulate the production of an important enzyme called as DHT which is involved in conversion of

\section{Corresponding author: Gopikumar MS,}

Associate Professor, department of Physiology, Xavier University School of Medicine, Aruba

No 5, Brebastraat, Ponton, Oranjestad, Aruba

Email: gshivaramaiahmd@xusom.com testosterone to DHT. DHT plays a very important role during early embryogenesis by its role in development and differentiation of male external genitalia. Deficiency of DHT during first trimester in the developing male fetus will impact the development of external genitalia leading to ambiguous genitalia in new borns.

46, XY DSD, that too specifically 5AR2D pose a major challenge for the parents and physicians of such individuals, as they must navigate the disorder, correctly diagnose it and manage it to preserve the quality of life. Every effort should be made to accurately diagnose a newborn with ambiguous genitalia. The diagnosis and care for patients with DSD involves a holistic approach and discussion among endocrinologists, surgeons, psychologists, social workers and family members. The degree of patients' masculinity and penis size, response to androgens and treatments, social culture and norms, and quality of life are considered in the care ${ }^{(1,2)}$.

Gender Ambiguity in 5AR2D: The earliest description of the 5AR2D and its surgical repair dates 
to before century, made by the Greek historian Diodorus Siculus. It describes Callo, who is supposedly a married female, who developed a tumor in the genital area. When a physician operated him, he discovered male genital parts with a functioning urethra, which he was able to bring to surface. Thus, the individual became a fully functioning male named Callon ${ }^{(3)}$.

In another study, a 21 year-old-woman, born out of a non-consanguineous marriage presented to physician with undeveloped breasts, ambiguous genitalia, deep voice, and primary amenorrhea. She had normal serum hormone profiles except for elevated total testosterone and an elevated testosterone to DHT ratio. Subsequently, on diagnosis with $5 \mathrm{AR} 2 \mathrm{D}$, she opted to remain as a female and underwent a surgery with hormonal replacement therapy using estrogen ${ }^{(4)}$.

In another study, six Arab subjects were evaluated for male pseudohermaphroditism. Out of six subjects, one subject was pubertal, 2 subjects were prepubertal and the remaining three were post pubertal. All the subjects were raised as females till the point of evaluation. One of the subjects was also married as a female. After their evaluation 2 subjects changed their gender to male, two remained as female and the other two subjects decided to take up a conservative female role and equivocal sex status. (5)

Genetics of 5ARD2: The rare 5AR2D was first described in 1974 in patients with pseudovaginal perineoscrotal hypospadias, micro phallus, and cryptorchid testes ${ }^{(6)}$. The condition is autosomal recessive and often found in males born in consanguineous parents in areas with high rates of inbreeding ${ }^{(7)}$. The major gene mutations in 5AR2D occur in the androgen receptor (AR) and steroid-5-alpha-reductase 2, (SRD5A2) genes (1). Type 1 isoenzyme is encoded by the SRD5A1 gene located on chromosome 5p15, and expressed mainly in the liver and non-genital skin ${ }^{(6)}$. Type 2 isoenzyme is encoded by the SRD5A2 gene on chromosome 2, band $\mathrm{p} 23$, and is expressed at high levels in the prostate, the epididymis, seminal vesicles, genital skin, and its efficiency leads to male pseudohermaphroditism, with incomplete differentiation of male genitalia ${ }^{(2)}$.

SRD5A2 was cloned and shown to contain five exons and four introns, which have over 65 known mutations, including point mutations, deletions, and insertions ${ }^{(1)}$. The most frequent polymorphism at exon 1, V89L, at the $89^{\text {th }}$ codon, results in valine to leucine substitution, and
Decreases 5-alpha-reductase 2 activity by approximately $30 \%$. V89L polymorphism is also more prevalent in patients with micropenis than in normal males ${ }^{(6,8)}$. Results of one major study of 33 subjects confirm the predominance of homozygous $(69.1 \%) v s$. compound heterozygous mutations $(30.9 \%)$, whereas deletions and disruptive mutations were relatively rare. Mutations was predominantly seen in exons $1(35.8 \%)$ and $4(21.7 \%)$, whereas exons $3(11.3 \%)$ and $5(9.4 \%)$ seemed to be $\operatorname{rare}^{(6,9)}$.

In another study involving 24 subjects with 5 alpha reductase 2 deficiency from Saudi Arabia born out of a consanguineous marriage. Direct sequencing of their SRD5A2 gene was done from their peripheral blood using PCR technique. Their gene analysis revealed 3 missense mutations (p.R246Q, p.P181L, p.A228T) in 11 subjects, one splice site mutation (IVS1-2A $>\mathrm{G}$ ) in 11 subjects and 2 nonsense mutations (p.R227X and p.R103X) in 2 subjects ${ }^{(10)}$.

In Spain, 146 index patients with 46, XY DSD were studied between 2002 and 2010. In these patients, gonads were confirmed as testes. SRD5A2 gene mutation was found in 9 index patients. One of the mutations (p.Y188CfsX9) has never been reported before, suggesting that there may be more mutations unrecognized yet ${ }^{(11)}$.

Epidemiology: The largest 5AR2D affected kindred known are New Guinean, Dominican and Turkish. The New Guinean kindred's mutation was the first group described, with deletion of the $5 \alpha$-reductase 2 gene of more than $20 \mathrm{~kb}$ resulting in a full loss of enzymatic activity. The Dominican kindred have a missense mutation in exon 5, substituting thymidine for cytosine and resulting in a substitution of tryptophan for arginine, resulting in reduction in binding of $5 \alpha$-reductase- 2 to its critical cofactor NADPH and a great decrease in enzymatic activity. The Turkish kindred have a single base deletion in exon 5 , causing a frame shift mutation with complete loss of enzymatic activity ${ }^{(7)}$.

5AR2D was also identified in populations not considered at risk of inbreeding, such as Europeans or North Americans, particularly in Quebec ${ }^{(12)}$.

Clinical Presentation: 5-alpha- reductase 2 deficiency is characterized by external female phenotype at birth, with a shallow vaginal pouch, hypospadias, which is an opening of the urethra on the underside of the penis, and a clitoral-like phallus. Presence of bilateral 
testes and normally developed internal male genitalia (7), but an underdeveloped prostate and a bifid scrotum. This is due to the inability to convert testosterone to dihydrotestosterone (DHT). DHT is responsible for the differentiation of genital tubercle and urogenital sinus into the prostate, urethra and external genitalia. Thus, male differentiation fails to occur despite high circulating testosterone levels ${ }^{(13,14)}$.

Gender Choice and Fertility: Most of the patients with 5 alpha reductase 2 deficiency are assigned female gender at birth and are raised as female child. At puberty, the surge in testosterone production prompts virilization, and enlargement of the genitalia with appearance of secondary sexual characteristics like development like muscle growth deepening of voice, pubic and axillary hair ${ }^{(1,7)}$. Lack of breast development and amenorrhea prompts the suspicion of diagnosis at puberty for most of the subjects with 5 alpha reductase 2 deficiency. ${ }^{(6,16)}$.

Fertility is a challenge due to low sperm production, defective transformation of spermatogonia into spermatocytes, and the inability to liquefy semen due to a lack of prostate specific antigen and seminal fluid ${ }^{(7)}$. Two known extremes of the phenotype are an arginine substitution for glycine at position 35, resulting in a female phenotype with minor virilization at puberty, and a serine substitution for a glycine at position 196 resulting in a predominantly male phenotype ${ }^{(15)}$. Other mutations lead to subtle abnormalities in the enzyme may underlie some forms of commonly encountered urogenital birth defect in males as well as androgendependent disorders such as male pattern baldness, acne, hirsutism, and benign or cancerous growth of the prostate $^{(14,15)}$.

Diagnosis: Traditionally, the diagnosis relies on DHT measurement, but the results being equivocal can sometimes lead to misdiagnosing the condition. An alternative approach for diagnosis of 5AR2D is urinary steroid profiling (USP), a readily available testing option. In one study, of the 15 patients undergoing USP, all showed low ratios in at least 2 of the 4 pairs of 5-alpha- and 5-beta-reduced steroid metabolites. USP is considered as an ideal test for biochemical phenotyping in 5AR2D 3 months after the birth of the child. ${ }^{(12,13)}$. Mutational analysis of SRD $5 A 2$ by PCR and direct DNA sequencing of all 46, XY DSD patients is the key to the diagnosis of 5AR2D, as this is the ultimate tool for diagnosis. Making use of this wonderful technology for identifying the chromosomal abnormalities of DSD will probably help to get a confirmed diagnosis ${ }^{(12,16)}$.

The biological diagnosis of 5AR2D is usually supported by an increase in the T/DHT ratio after human chorionic gonadotropin (HcG) stimulation testing, which is first line of diagnosis in infants and pre-pubertal children ${ }^{(1,6)}$. In some cases, however, the diagnosis cannot be ruled out by if there is no evidence of elevated T/DHT ratio after HCG stimulation. ${ }^{6,13)}$ T/DHT ratio greater than 8.5 is suggestive of the deficiency and these babies and infants should be investigated further with molecular analysis of the 5 -alpha-reductase-2 gene ${ }^{(12)}$.

In another major study, over $72 \%$ of patients presented a ratio above 10 , confirming that this ratio is a good indicator and could easily be used for screening patients ${ }^{(6)}$. Adult subjects deficient in 5 $\alpha$-reductase- 2 activity also have elevated T/DHT ratios with normalto-elevated testosterone levels compared with normal subjects. What distinguishes them from androgeninsensitive subjects who may also have abnormal $\mathrm{T} /$ DHT ratios is abnormal ratios of $5 \beta$ - to $5 \alpha$-reduced glucocorticoid and other steroid metabolites, indicating that this condition is a generalized defect in hepatic steroid $5 \alpha$ - metabolism ${ }^{(1,17)}$.

The definitive diagnosis of mutation causing 5AR2D can now be made by using special techniques like complementary DNA analysis by using the biopsy material of the tissues, peripheral blood and cultures obtained from fibroblasts ${ }^{(7,18)}$

Treatment options in 5AR2D: Early diagnosis of 5AR2D is a key factor in its treatment. Early diagnosis allows the children to be raised as males at an early age and children can avoid embarrassment of gender conversion at a later age. In an interesting case study, two siblings, 3 years apart, both undergoing DHT treatment were successful in increasing phallic size. The siblings were born to Pakistani parents who were first cousins and practising Muslims. When the first child was born, early diagnosis and management of 5AR2D encouraged them to raise this baby as a male in accordance with the chromosomal sex. The younger sibling was born just when the older one was being diagnosed. ${ }^{(1)}$.

DHT gel treatment for pediatric micropenis was studied with 76 pediatric patients with 46, XY DSD, who were treated with DHT gel (0.1-0.3 mg/kg/day) for three to six months. 22 of these patients had SRD5A2 mutations. The penis length of the patients significantly improved with DHT treatment. The length of the penis 
increased significantly after long term treatment with DHT resulting in greater improvement. Therefore, the study shows that local application of DHT gel can promote penis growth effectively without systemic adverse reactions ${ }^{(1)}$.

Often these individuals require long-term psychological support to aid in making an informed decision. If the parents chose to raise a female, gonadectomy and surgical correction of the external genitalia are indicated to avoid masculinization, with vaginoplasty for a healthy sexual life. An estrogen-only hormone replacement therapy must be administered these patients throughout life until the 50th year of age ${ }^{(3)}$. In New Guinea, where tribal culture is gender-segregated with very traditional male and female roles, rituals and rules, the upbringing and transition from female to male is very difficult for 5AR2D individuals ${ }^{(7)}$.

\section{Conclusion}

The multiples studies on 5AR2D underline the wide spectrum of phenotypes and biological profiles in patients with the condition. So far, no genotype- phenotype relationship could be determined ${ }^{(6)}$. Diagnosis is made by a combination of criteria such as DSD, virilization at puberty, and a marked increase in the T/DHT ratio after HCG testing. Due to often false negatives, DNA sequencing of the entire SRD5A2 gene is necessary. Physicians must be informed and aware of DSD and 5AR2D in particular, and make informed clinical decisions with the family of the affected individuals, due to the often-sensitive matter in a variety of communities and societies. 5AR2D should be considered in all XY newborns with ambiguous genitalia and normal or high plasma testosterone secretion ${ }^{(9)}$. The decisions made should consider the fact that at puberty, the individuals often become masculine and identify as males. Also, management decisions should aim at preserving functionality, quality of sexual and day-to-day life.

Ethical Clearance: Since this is a review article, we did not need approval from the Institutional review board, although the board was aware that we are into this article.

\section{Conflict of Interest: Nil}

Source of Funding: Self.

\section{References}

1. Fu X, Zhang W, Qu X. Correlation of androgen receptor and SRD5A2 gene mutations with pediatric hypospadias in 46, XY DSD children. Genetics and Molecular Research. 2016; 15(1).

2. Odame I, Donaldson M, Wallace A, Cochran W, Smith P. Early diagnosis and management of 5 alpha-reductase deficiency. Archives of Disease in Childhood. 1992;67(6):720-723.

3. Markantes G, Deligeoroglou E, Armeni A, Vasileiou V, Damoulari C, Mandrapilia A et al. Callo: The first known case of ambiguous genitalia to be surgically repaired in the history of Medicine, described by Diodorus Siculus. HORMONES. 2015;

4. Maleki N, Kalantar Hormozi M, Iranparvar Alamdari M, Tavosi Z. 5-Alpha-Reductase 2 Deficiency in a Woman with Primary Amenorrhea. Case Reports in Endocrinology. 2013;2013:1-4.

5. Al-Attia H. Male pseudohermaphroditism due to 5 alpha-reductase-2 deficiency in an Arab kindred. Postgraduate Medical Journal. 1997;73(866):802807.

6. Maimoun L, Philibert P, Cammas B, Audran F, Bouchard P, Fenichel P et al. Phenotypical, Biological, and Molecular Heterogeneity of 5 $\alpha$-Reductase Deficiency: An Extensive International Experience of 55 Patients. The Journal of Clinical Endocrinology \& Metabolism. 2011;96(2):296-307.

7. Kang H, Imperato-McGinley J, Zhu Y, Rosenwaks $Z$. The effect of $5 \alpha$-reductase- 2 deficiency on human fertility. Fertility and Sterility. 2014;101(2):310316.

8. Sasaki G, Ogata T, Ishii T, Kosaki K, Sato $\mathrm{S}$, Homma $\mathrm{K}$ et al. Micropenis and the $5 \alpha$-Reductase-2 (SRD5A2) Gene: Mutation and V89L Polymorphism Analysis in 81 Japanese Patients. The Journal of Clinical Endocrinology \& Metabolism. 2003;88(7):3431-3436.

9. Maimoun L, Philibert P, Cammas B, Audran F, Pienkowski C, Kurtz $\mathrm{F}$ et al. Undervirilization in $\mathrm{XY}$ newborns may hide a $5 \alpha$-reductase deficiency: report of three new SRD5A2 gene mutations. International Journal of Andrology. 2010;33(6):841-847.

10. Alswailem M, Alzahrani O, Alghofaili L, Qasem E, Almohanaa M, Alsagheir A et al. Molecular genetics and phenotype/genotype correlation of 5- $\alpha$ reductase deficiency in a highly consanguineous 
population. Endocrine. 2018;63(2):361-368.

11. Fernández-Cancio M, Audí L, Andaluz P, Torán N, Piró C, Albisu $\mathrm{M}$ et al. SRD5A2 gene mutations and polymorphisms in Spanish 46,XY patients with a disorder of sex differentiation. International Journal of Andrology. 2011;34(6pt2):e526-e535.

12. Chan A, But B, Lee C, Lam Y, Ng K, Tung J et al. Diagnosis of 5 -Reductase 2 Deficiency: Is Measurement of Dihydrotestosterone Essential?. Clinical Chemistry. 2013;59(5):798-806.

13. Al-Jurayyan N. Ambiguous Genitalia: Two Decades of Experience. Annals of Saudi Medicine. 2011;31(3):284-288.

14. Akcay T, Fernandez-Cancio M, Turan S, Güran T, Audi L, Bereket A. ARandSRD5A2gene mutations in a series of 51 Turkish 46,XY DSD children with a clinical diagnosis of androgen insensitivity. Andrology. 2014;2(4):572-578.

15. Thigpen A, Davis D, Milatovich A, Mendonca B, Imperato-McGinley J, Griffin $\mathrm{J}$ et al. Molecular genetics of steroid 5 alpha-reductase 2 deficiency. Journal of Clinical Investigation. 1992;90(3):799809.

16. Deeb A, Suwaidi H, Ibukunoluwa F, Attia S. Phenotype, Sex of Rearing, Gender Re-Assignment, and Response to Medical Treatment in Extended Family Members with a Novel Mutation in the SRD5A2 Gene. Journal of Clinical Research in Pediatric Endocrinology. 2016;8(2):236-240.

17. Fernández-Cancio M,Nistal M, Gracia R, MolinaM, Tovar J, Esteban C et al. Compound Heterozygous Mutations in theSRD5A2Gene Exon 4 in a Male Pseudohermaphrodite Patient of Chinese Origin. Journal of Andrology. 2004;25(3):412-416.

18. Ng W, Taylor N, Hughes I, Taylor J, Ransley P, Grant D. 5 alpha-reductase deficiency without hypospadias. Archives of Disease in Childhood. 1990;65(10):1166-1167. 